\title{
THz quantum cascade lasers based on a hyperuniform design
}

R. Degl'Innocenti, Y.D. Shah, L. Masini, A. Ronzani, A. Pitanti, Y. Ren, D.S. Jessop, A. Tredicucci, H.E. Beere, D.A. Ritchie

Published in Quantum Sensing and Nanophotonic Devices XII, edited by Manijeh Razeghi, Eric Tournié, Gail J. Brown, Proc. of SPIE v. 9370, 93700A

(C) 2015 Society of Photo-Optical Instrumentation Engineers. One print or electronic copy may be made for personal use only. Systematic reproduction and distribution, duplication of any material in this paper for a fee or for commercial purposes, or modification of the content of the paper are prohibited. 


\title{
THz Quantum Cascade Lasers based on a hyperuniform design
}

\author{
R. Degl'Innocenti*a, Y. D. Shaha, L. Masini ${ }^{\mathrm{b}}$, A. Ronzani ${ }^{\mathrm{b}}$, A. Pitantib, Y. Ren ${ }^{\mathrm{a}}$, D. S. Jessop ${ }^{\mathrm{a}}$, A. \\ Tredicuccic ${ }^{\mathrm{c}}$ H. E. Beere ${ }^{\mathrm{a}}$, D. A. Ritchie ${ }^{\mathrm{a}}$ \\ ${ }^{a}$ Cavendish Laboratory, University of Cambridge, J. J. Thomson Avenue, Cambridge CB3 0HE, \\ United Kingdom; ${ }^{b}$ NEST, Istituto Nanoscienze - CNR and Scuola Normale Superiore, Piazza San \\ Silvestro 12, Pisa, I-56127, Italy; 'Dipartimento di Fisica "E. Fermi” Universita' di Pisa, Largo \\ Pontecorvo 3, 56127 Pisa, Italy
}

\begin{abstract}
A terahertz quantum cascade laser has been realized from an isotropic disordered hyperuniform design. Such a system presents a photonic band-gap although it is characterized by an efficient depletion of the long range order. Hyperuniform patterns allow greater versatility in engineering band gaps in comparison to standard photonic-crystal materials. Bidimensional hyperuniform patterns were simulated for hexagonal tiles composed of high refractive index disks merged in a low dielectric constant polymeric matrix. Based on this design, quantum cascade lasers were fabricated by standard photolithography, metal evaporation, lift-off and dry-etching techniques in a half-stack bound to continuum active region emitting around $2.9 \mathrm{THz}$.
\end{abstract}

Keywords: Quantum cascade lasers, terahertz, hyperuniform disordered distribution, photonic band gap

\section{INTRODUCTION}

Terahertz (THz) quantum cascade lasers (QCLs) are commonly employed in a huge variety of applications, ranging from metrology ${ }^{1}$, sensing ${ }^{2}$ and spectroscopy ${ }^{3}$. Most of these applications require sources with an excellent spatial profile, and a precise spectral control of the emission profile. Consequently, the research has been focused in the development of distributed feedback grating (DFB) $)^{4,5}$, or alternatively on two-dimensional photonic crystal (PC) $)^{6,7}$ designs. While periodic photonic systems have been thoroughly investigated, research into photonic band gap (PBG) materials has not been fully exploited to date. Aperiodic photonic systems, such as quasi crystals ${ }^{8,9}$, offer a higher level of complexity, such as rotation symmetries prohibited by conventional periodic systems, and a larger degree of freedom given by a richer Fourier spectrum. Even though quasi-crystals lack translational symmetry, they still present long-order range and a quasiperiodicity. These features can be used for realizing non conventional laser cavities, such as a Penrose lattice arrangement ${ }^{10}$. An alternative concept is the random laser ${ }^{11}$, which relies instead on the creation of scattering paths within a disordered laser active material. Even more intriguing is the possibility of engineering isotropic disorder in a material where the long-range order is suppressed but still the distribution exhibits a photonic band gap. These PBGs can be tuned by acting on an order parameter which regulates the distribution properties. The work presented here is based on this latter approach. In particular, a hyperuniform distribution pattern was investigated, which is characterized by the total suppression of density fluctuations for long wavelengths ${ }^{12,13}$. An active region design emitting at $2.9 \mathrm{THz}$, was processed into a disordered hyperuniform distribution of pillars, exhibiting a photonic band gap as large as $18 \%$ approaching the band gap of a conventional PC. The device lases on a localized mode at the lower edge of the band gap, which has been scaled in order to match the gain profile of the active region, representing the first demonstration of lasing action in such a disordered structure.

\section{THEORETICAL BACKGROUND AND SIMULATIONS}

The procedure for the realization of a 2D hyperuniform pattern, according to Ref. [12] is based on a 2D scatterers distribution wherein the density fluctuations grows more slowly than the sampling window itself. In reciprocal space, this corresponds to a structure factor $\mathrm{S}(\mathrm{k})$ that tends to zero for wavenumbers $\mathrm{k}$ that tend to zero. The structure factor $\mathrm{S}(\mathrm{k})$ is defined in Eq. 1:

*rd448@cam.ac.uk

Quantum Sensing and Nanophotonic Devices XII, edited by Manijeh Razeghi, Eric Tournié, Gail J. Brown, Proc. of SPIE Vol. 9370, 93700A - (C) 2015 SPIE · CCC code: 0277-786X/15/\$18 · doi: 10.1117/12.2083678 


$$
S(k)=\left|\sum_{i=1}^{N} \exp \left(i k r_{i}\right)\right|^{2}
$$

where $\mathrm{N}$ is the total number of scatterers in the distribution and $\mathrm{r}_{\mathrm{i}}$ the scatterer positions in direct space. The global properties of the distribution are isotropic while locally the system is anisotropic. Alternatively, a hyperuniform system can be characterized by an efficient depletion of the long range interactions. In particular, an interesting subclass of hyperuniform distributions was chosen, which is isotropic, disordered and characterized by a structure factor $\mathrm{S}(\mathrm{k})$ exactly
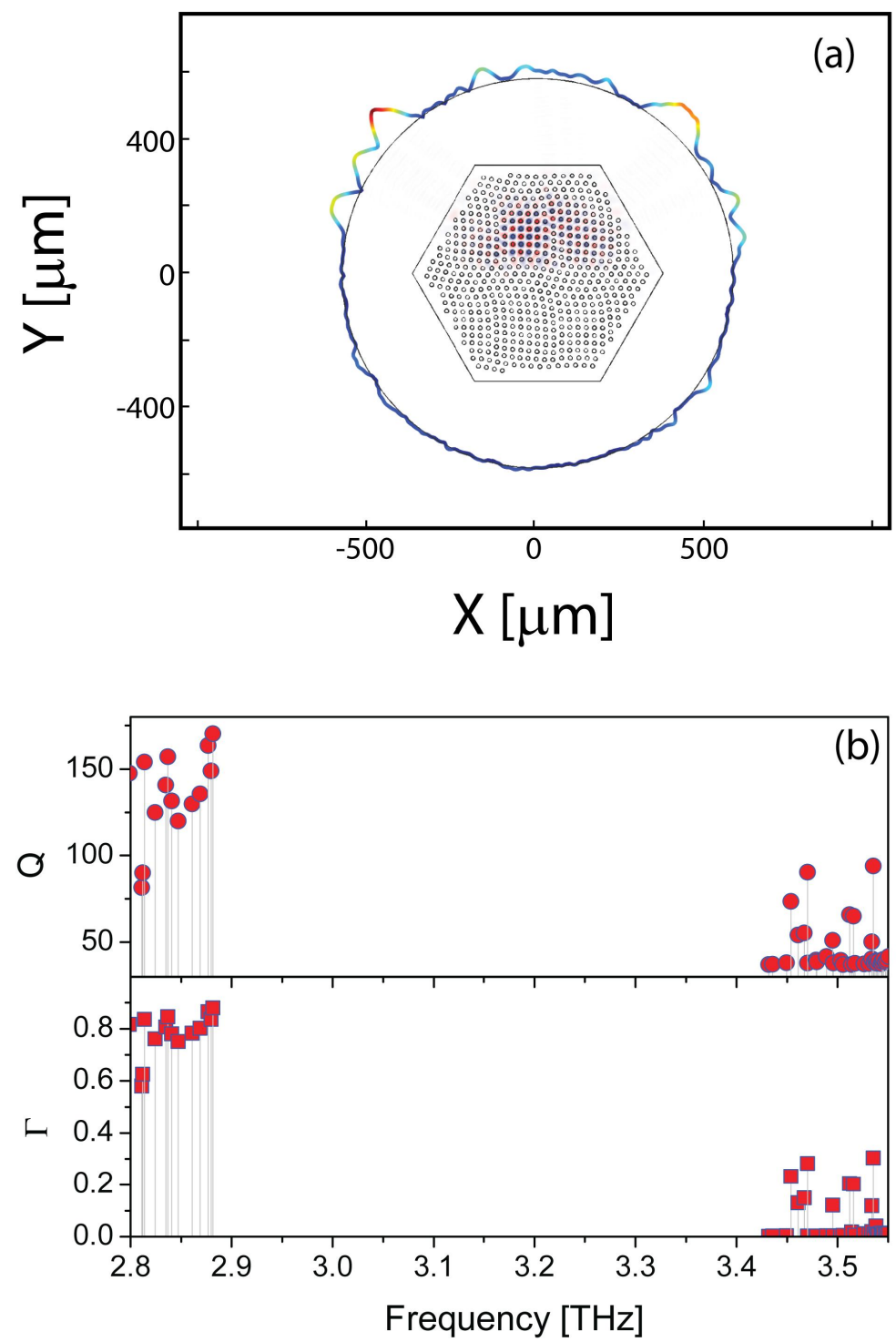

Figure 1 a) 2D finite element simulations performed with the commercial software Comsol Multiphysics of a hyperuniform pattern with an order parameter $\chi=0.7$ of disks with refractive index $n=3.55$ merged in a low dielectric medium with $n=$ $1.58+i^{*} 0.0087$, simulating the active region and the Cyclotene resin, respectively. The surface represents the Electric-field of the optical mode while the line corresponds to the far-field normalized, being the deformation proportional to the intensity. The mode has an eigenfrequency of $2.87 \mathrm{THz}$, and is the one with the highest $\mathrm{Q}$ factor on the lower edge of the photonic band-gap. b) $\mathrm{Q}$ factors and confinement factor $\Gamma$ of the modes supported by this structures.

equal to zero for $\mathrm{k}$ lower than a critical value $\mathrm{k}_{\mathrm{c}}$. In this case, it is more convenient to define a parameter $\chi$ which represents the fraction of wavenumbers $\mathrm{k}$ in the Brillouin zone that are set to zero. The distribution is obtained by depleting a region in the k-space with a total number of $\mathrm{M}(<\mathrm{N})$ scatterers and the $\chi$ parameter is consequently calculated 
as $\mathrm{M} / \mathrm{N}$, thus varying between 0 and 1 . The higher the $\chi$ factor the higher the degree of hyperuniformity. For sufficiently high $\chi$ the system develops long-range translational order, and the system evolves towards the standard photonic crystal periodic arrangement. On the other side, for low $\chi$ values the system does not present a PBG. This peculiarity, the presence of a PBG which is normally associate to long-range order, in this kind of disordered distribution is counterintuitive and it represents one of the most fascinating feature of this approach. The PBG size increases with the level of hyperuniformity and short-range geometric order (the variance in the link length between a scatterer and its first neighbors) of the system. The interaction between these two effects with the Mie resonances of the scatterers for optimal disk radii and links gives rise to the formation of a PBG. The hyperuniform pattern was generated within the first Brillouin zone of a triangular lattice of disks. This lattice distribution based on high index scatterers in a low index background was preferred since it yields in conventional PC devices the largest PBG for TM modes, as required by the selection rules for THz QCLs. A distribution characterized by an order parameter $\chi=0.7$ was chosen since it offers a trade-off between having localized modes and a sufficiently large overlap of the mode with the active region (AR). The final design consists of a hyperuniform distribution of disks arranged in a hexagonal tile. This pattern was transferred in a finite element method solver (Comsol Multiphysics v4.2), where the eigenmodes are simulated using the effective index approximation, in order to simulate the real device, as shown in Fig. 1 a). The 2D hexagonal distribution consists of disks, with refractive index $\mathrm{n}$ of 3.55 , diameter of $11.5 \mu \mathrm{m}$ and a relative distance of $\sim 25 \mu \mathrm{m}$. These pillars are surrounded by a dielectric polymeric matrix, simulating the resin Cyclotene, with $\mathrm{n}=1.58+\mathrm{i}^{*} 0.0087$.
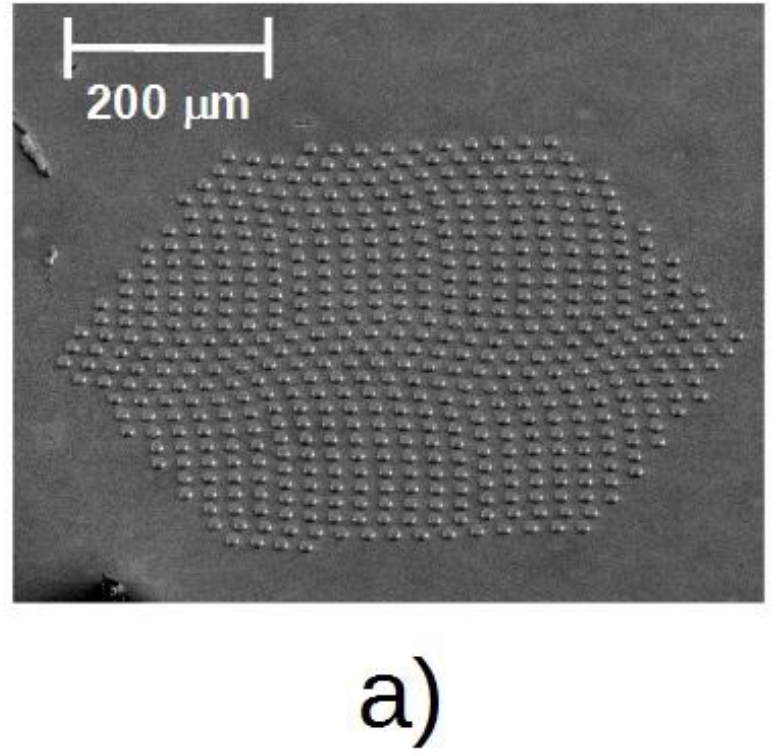
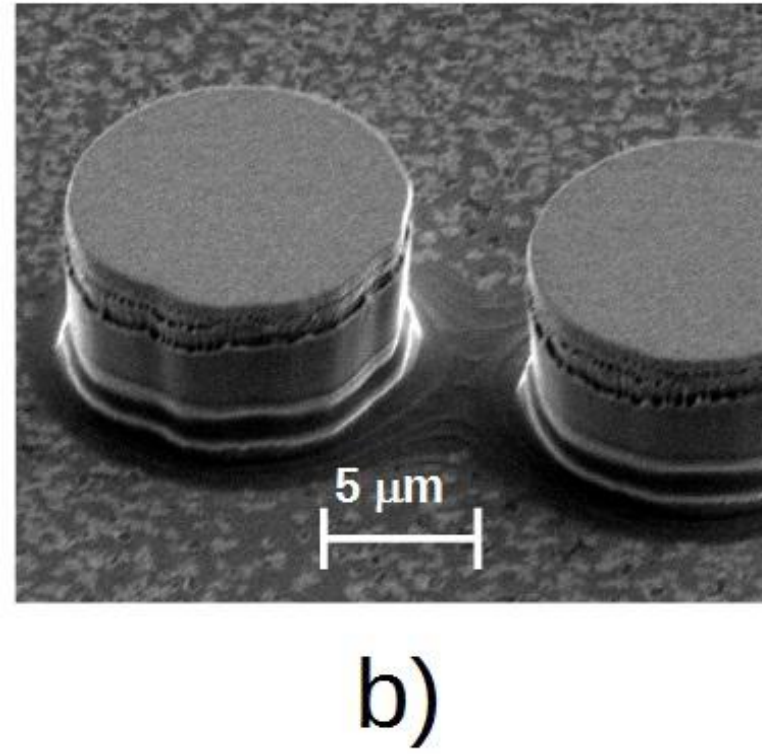

Figure 2 a) Scanning electron microscope picture (SEM) of the $6.55 \mu \mathrm{m}$ high pillar distribution. The highest Q factor modes were frequency centered around $2.9 \mathrm{THz}$ in order to match the gain curve of the active region. The 2D pattern was etched through the active region with a reactive ion etching process by using metal masking; b) detailed picture of the etching profile. Even though it is possible to observe a re-deposition of the material on the pillar' sides, the sidewalls are nominally vertical.

The polymer Cyclotene (3022-46 from Dow Company) was chosen as the low refractive index material, because of its low losses in the terahertz range, good thermal and mechanical properties and its previously successful use in the fabrication of conventional photonic crystal THz QCLs ${ }^{6}$. The eigenfrequency analysis reveals the opening of a TM photonic bandagap as large as $18 \%$ of the central frequency, as shown in Fig. $1 \mathrm{~b}$ ). The modes on the lower edge of the gap, centered around $2.87 \mathrm{THz}$, have a maximum of the E-field in the active region pillars, with a confinement factor of $\sim 80 \%$, as shown in Fig. 1 b). The confinement factor is evaluated through the overlap integral $\Gamma$, which is defined as the integral of the optical mode electric energy density within the active region, normalized to the same integral calculated over the whole space, as reported in Eq. 2, where $\varepsilon$ is the dielectric constant;

$$
\Gamma=\iint_{x y=A R} \varepsilon(x, y)|E(x, y)|^{2} / \iint_{x y=\infty} \varepsilon(x, y)|E(x, y)|^{2}
$$


Among the many optical modes supported by this structure, the localized ones at the lower border of the band edge emerge with a higher $\mathrm{Q}$ factor $>170$, as shown in Fig. $1 \mathrm{~b}$ ). It is assumed that lasing action will take place on these modes because of the higher $\mathrm{Q}$ factor. The modes on the upper edge of the PC gap have a node passing through the pillars, and therefore a lower overlap integral $\Gamma$ as shown in Fig. 3 b).

\section{DEVICE FABRICATION}

A $6.55 \mu \mathrm{m}$ thick active region based on a bound to continuum design and emitting around $2.9 \mathrm{THz}$ has been used for the device fabrication. It is a repetition of the bound to continuum design reported in Ref. 14 but with only 45 repetitions. Reactive-ion-etching (RIE) process allowed the fabrication of pillars with vertical sidewall in half stack active region by using metal mask. The etching of more powerful, full stack active region into pillars with acceptable vertical sidewall would require the use of a different equipment; an inductive coupled plasma (ICP) RIE. The active region was wafer bonded using Au-Au thermocompressive bonding on $\mathrm{n}^{+} \mathrm{GaAs}$ substrate. The hyperuniform pattern of metal (Ti/Au 7/250 $\mathrm{nm}$ ) disks was transferred on the top surface of the active region through standard lithography, thermal evaporation and lift-off. This pattern was then etched through the whole active region using a reactive ion etching process, thus realizing a 3D hyperuniform patterns of pillars. The RIE process used a gas mixture of 4:10 $\mathrm{SiCl}_{4}$ : $\mathrm{Ar}$, a pressure of $25 \mathrm{mTorr}$, a power of $100 \mathrm{~W}$, temperature of $25^{\circ} \mathrm{C}$ for a total etching time of 22 minutes. Fig. 2 a) shows the pillars patterns transferred in the active region and b) a detail of the etching profile. The next critical step was the spinning and processing of a Cyclotene (BCB) layer to achieve an efficient surface planarization. The BCB layer is spun at $4000 \mathrm{rpm}$ and then thermally cured up to $265^{\circ} \mathrm{C}$ for two hours with the final result to completely cover the top of the pillars as shown in Fig. 3 a). In order to uncover the top of the pillars to allow the top contact metal evaporation, the BCB layer was etched using a Plasma-Pod RIE machine from JLS Designs, with $75 \mathrm{~W}$ power, a mixture $(60 \%-40 \%)$ of $\mathrm{CHF}_{3} / \mathrm{O}_{2}$ and a pressure of $60 \mathrm{sccm}$ for 3 minutes. The pillars head are then exposed as shown in Fig. $3 \mathrm{~b}$ ). As a final step of lithography, metal $(\mathrm{Ti} / \mathrm{Au} \mathrm{10/100} \mathrm{nm})$ thermal evaporation and lift-off is needed for the realization of the top contact. The final device is then mounted onto a copper block, and wire bonded.
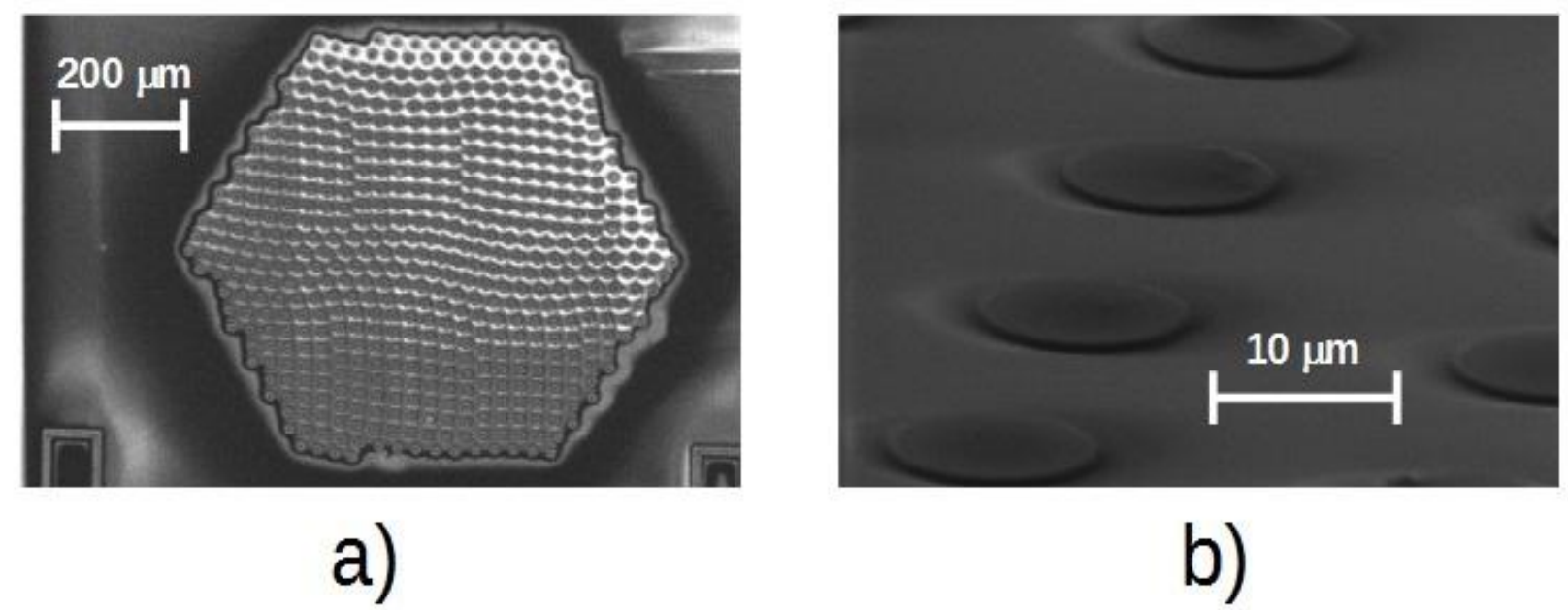

Figure 3 a) SEM picture of the pillars completely covered after spinning and curing the BCB layer. b) SEM picture of the pillars' heads exposed after having dry etched the sample with a $\mathrm{CHF}_{3} / \mathrm{O}_{2}$ mixture in a reactive etching machine. This is a critical step which is required in order to define a metallic top contact layer and to bias the device.

\section{MEASUREMENTS}

The lasers were tested on a cold-finger cryostat in pulsed operation mode with a repetition rate of $100 \mathrm{kHz}$ and a duty cycle of $20 \%$, with the power measurements being taken by a Golay cell and lock-in amplifier. The light was collected using parabolic mirrors with $7 \mathrm{~cm}$ focal length and then focused on the Golay with a second parabolic mirror having focal length of $10 \mathrm{~cm}$. The electronic transport and power characteristics are compatible with the previous results for this active region and are shown in Fig. 4 a). The device shows lasing action only up to $35 \mathrm{~K}$, as reported in Fig. 4 b), instead of the $85 \mathrm{~K}$ reported for standard double metal QCL fabricated from this active region operating with $5 \%$ duty cycle. This performance reduction can be attributed to several detrimental factors. The non-ideal circular geometry and size of 
the final pillars, as shown also in Fig. 2 b), is considered to be the principal cause together with the lateral surface roughness. The discrepancy between real and simulated structures can be attributed to minor imperfections in the metallic disk geometry during the first lithographic step, which is then propagated in the etching process, such that the final device did not lase on the maximum of the gain curve. A non uniform top contact evaporation and the wire bonding are other critical factors which could affect the final performance of the device. The low power emission did not allow far-field measurements to be taken. Thicker and more powerful active region design could be employed yielding higher power level. The fabrication of such structures, with typical thickness of $\sim 12 \mu \mathrm{m}$, requires the use of inductive-coupled RIE, which would also help in improving the quality of the etching, in terms of vertical sidewall profiles and roughness. However, the aim of this work was to demonstrate lasing in an unconventional cavity design.

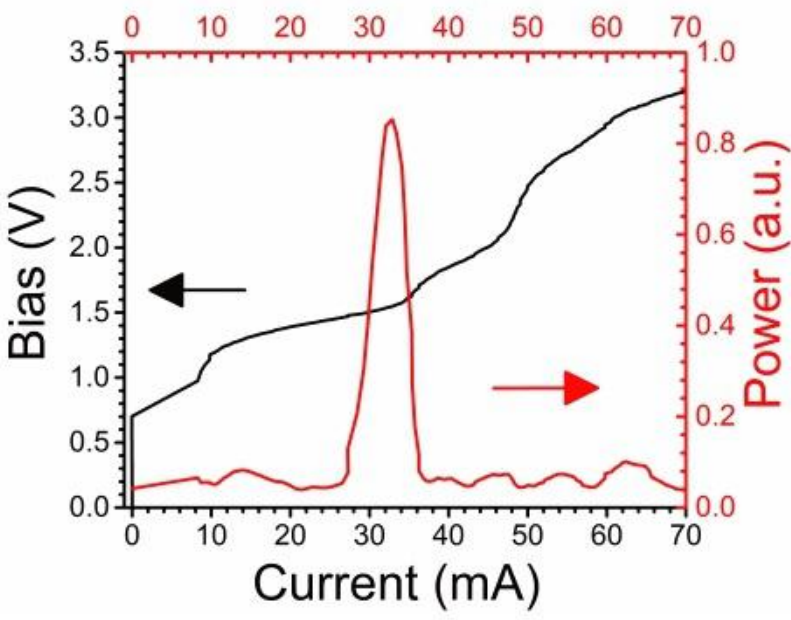

a)

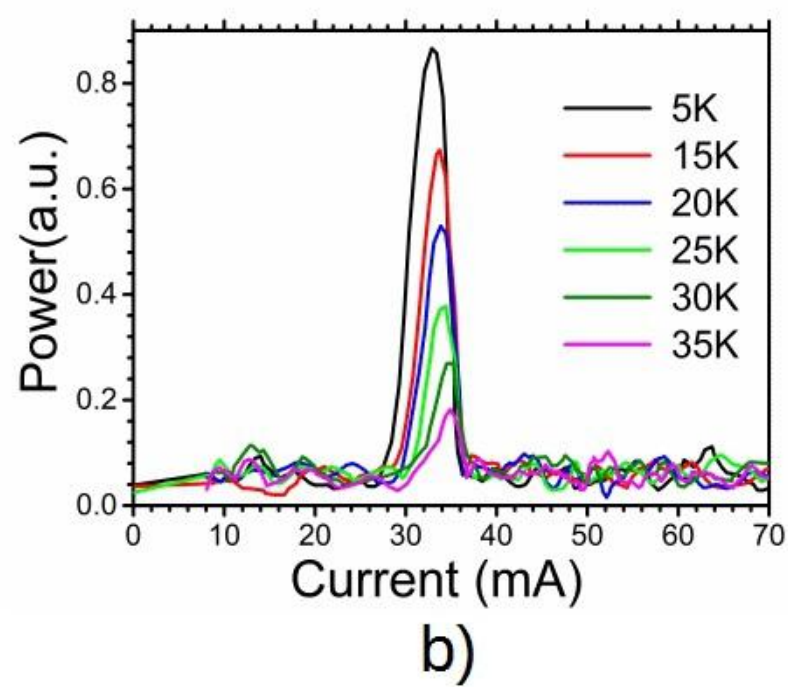

Figure 4 a) Voltage-bias-power characteristic curves of the device for $100 \mathrm{kHz}$ repetition rate, $20 \%$ duty cycle, $4 \mathrm{~K}$ temperature. Lasing occurs for bias compatible with the reported curves for this active region. b) The maximum lasing temperature reported is $35 \mathrm{~K}$.

\section{CONCLUSIONS}

In conclusion, we have demonstrated the first realization of lasing action in an isotropic, disordered hyperuniform distribution. This result has been accomplished by realizing a THz QCL based on a pillars distribution etched in a 2.9 $\mathrm{THz}$ emitting active region. A protocol for the design of hyperuniform distributions was successfully developed and the final pattern was investigated and simulated with finite element method analysis. Simulations and measurements confirm lasing action in localized optical mode located at the bottom of the photonic band gap. This result paves the way to the realization of lasers based on this concept presenting different degree of hyperuniformity, thus different mode localization and consequently far-fields and power emissions. This opens the path to non-conventional mode engineering with respect to conventional PC and quasi-crystal lasers.

\section{REFERENCES}

[1] Bartalini, S., Consolino L., Cancio, P., De Natale, P., Bartolini, P., Taschin, A., De Pas, M., Beere, H. E., Ritchie, D. A., Vitiello, M. S., Torre, R., "Frequency-Comb-Assisted Terahertz Quantum Cascade Laser Spectroscopy," Phys. Rev. X 4, 021006 (2014).

[2] Consolino, L., Bartalini, S., Beere, H. E., Ritchie, D. A., Vitiello, M. S. and De Natale, P., "THz QCL-Based Cryogen-Free Spectrometer for in Situ Trace Gas Sensing," Sensors 13(3), 3331-3340 (2013).

[3] Ren, Y., Hovenier, J. N., Higgins, R., Gao, J. R., Klapwijk, T. M., Shi, S. C., Klein, B., Kao, T.-Y., Hu, Q. and Reno, J. L., "High-resolution heterodyne spectroscopy using a tunable quantum cascade laser around 3.5 THz," Appl. Phys. Lett. 98, 231109 (2011). 
[4] Mahler, L., Koehler, R., Tredicucci, A., Beltram, F., Beere, H. E., Linfield, E. H., Ritchie, D. A. and Davies, A. G., "Single-mode operation of terahertz quantum cascade lasers with distributed feedback resonators," Appl. Phys. Lett. 84, 5446-5448 (2004).

[5] Fan, J. A., Belkin, M. A., Capasso, F., Khanna, S., Lachab, M., Davies, A. G. and Linfield, E. H., "Surface emitting terahertz quantum cascade laser with a double-metal waveguide," Opt. Express 14(24), 11672-11680 (2006).

[6] Zhang, H., Dunbar, L. A., Scalari, G., Houdre, R. and Faist, J. "Terahertz photonic crystal quantum cascade lasers," Opt. Express 15(25), 16818-16827 (2007)

[7] Chassagneux, Y., Colombelli, R., Maineult, W., Barbieri, S., Beere, H. E., Ritchie, D. A., Khanna, S. P., Linfield, E. H. and Davies, A. G., "Electrically pumped photonic-crystal terahertz lasers controlled by boundary conditions," Nature, 457, 174-178 (2009).

[8] Mahler, L., Tredicucci, A., Beltram, F., Walther, C., Faist, J., Beere, H. E., Ritchie, D. A. and Wiersma, D., "Quasi-periodic distributed feedback laser," Nature Photonics 4, 165169 (2010).

[9] Valy Vardeny, Z., Nahata, A., Agrawal, A., "Optics of photonic quasicrystals," Nature photonics 7, 177-187 (2013)

[10] Notomi, M., Suzuki, H., Tamamura, T. and Edagawa, K., "Lasing action due to twodimensional quasiperiodicity of photonic quasicrystal with a Penrose lattice," Phys. Rev. Lett. 92,123906 (2004).

[11] Wiersma, D. S., "Disordered photonics,", Nature Photonics 7, 188-196, (2013).

[12] Florescu, M., Torquato, S., Steinhardt, P. J., "Designer disordered materials with large, complete photonic band gaps," PNAS, 106(49), 20658-20663 (2009).

[13] Man, W., Florescu, M., Matsuyama, K., Yadak, P., Nahal, G., Hashemizad, S., Williamson, E., Steinhardt, P., Torquato, S. and Chaikin, P., "Photonic band gap in isotropic hyperuniform disordered solids with low dielectric contrast" Opt. Express 21(17), 19972-19981 (2013).

[14] Barbieri, S., Alton, J., Beere, H. E., Fowler, J., Linfield, E. H. and Ritchie, D. A., "2.9 THz quantum cascade lasers operating up to $70 \mathrm{~K}$ in continuous wave," Appl. Phys. Lett. 85, 1674 (2004). 